\title{
EVALUATION OF SURFACE ROUGHNESS PARAMETERS (Ra, Rz) IN DRILLING OF MDF COMPOSITE PANEL USING BOX-BEHNKEN EXPERIMENTAL DESIGN (BBD)
}

\author{
Prakash $\mathbf{S}^{1}$, Palanikumar $\mathrm{K}^{2}$, Lilly Mercy $\mathrm{J}^{3}$, Nithyalakshmi $\mathbf{S}^{3}$ \\ 1, 3, 4 Department of Mechanical \& Production Engineering, Sathyabama University, \\ ${ }^{2}$ Sri Sai Ram Institute of Technology, Chennai -600 044
}

\section{Abstract}

Medium Density fiberboard (MDF) panels are economic alternative to engineering materials because of their superior advantages in furniture industry. The degree of surface roughness of the MDF panel plays an important role, since any surface irregularities will reduce the final quality of the product. Design of experiments has been used to study the effects of the drilling parameters such as speed, feedrate, and drill diameter (input data's) used on the prediction of surface roughness (output response) on drilling MDF composite. The present work describes the development of mathematical models to predict the two surface roughness parameters namely average roughness( $\mathrm{Ra})$,and mean peak to valley height $(\mathrm{Rz})$ which were used to determine quantative surface characteristics of the panels. Experiments were performed under different drilling conditions of spindle speed, feed rate and drill diameter. Residual plots were constructed to analyze the variation between the experimental values and model values. Analysis of Variance (ANOVA) was employed to identify the level of importance in drilling parameters on their performance characteristics.

The effect of these parameters on surface roughness has been investigated using Box-Behnken experimental design. Response contours were constructed for determining the optimum drilling condition for the required surface roughness. The developed model establishes a correlation between speed, feed rate and drill diameter that influence the surface roughness in a MDF panel. Analysis of variance (ANOVA) is employed to study the surface roughness characteristics in drilling operation of MDF board. The analysis of variance showed high coefficient of determination $\left(R^{2}\right)$ value of 0.99 and 0.9842 for $R a$ and $R z$ respectively, thus ensuring a satisfactory adjustment of the second order regression model with experimental data. The verification experiment is carried out to check the validity of the developed model. The surface roughness of the predicted model during the confirmatory test has $2.06 \%$ and $2.631 \%$ error for $\mathrm{Ra}$ and $\mathrm{Rz}$ respectively, which is well within the tolerable limits.

Keywords: Analysis of Variance (ANOVA), Box-Behnken design, Contour Plots, Drilling, MDF panels, Response Surface Methodology

\section{INTRODUCTION}

Wood composites industry, including particle board and MDF panels has an important share within overall forest products sector. MDF is one of the most rapidly growing composite board products available in the marketplace. MDF is experiencing increased application in many product areas such as furniture, kitchen cabinets, and ready-to-assemble (RTA) furniture. It is made out of wood waste fibers glued together with resin by heat and pressure. Nowadays the MDF products are preferred over solid waste in many applications due to certain comparative advantages and mainly used in furniture industry. Painting, grain printing, and overlaying the new generation of lightweight papers are also enhanced by a high-density panel surface. The homogeneous core of MDF makes it especially suitable for embossing, molding, and general machining. A uniform density throughout the panel thickness results in better edge-fastening properties. The density profile is correlated to performance characteristics. Surface finish is an important parameter in manufacturing engineering, which can influence the performance of final parts and production.

Metal drilling and turning had been studied extensively in the literature, but MDF drilling has not received much attention. However, many works of various authors [1-6] represent the machining of MDF and other related wood composites. They strongly recommended that the machinability is dependent on the mechanism of cutting tool and work piece material.

From the literature, it has been asserted that machining MDF is strongly dependent on the machining 
parameters. Philbin and Gordon [7] studied the application of PCD tool in machining MDF. According to his study, the friction on the rake is small and the pressure exerted by uncut chip on the rake face mainly dominates the force on the rake face. Lin et al [8] reports about the machinabilty of MDF. These authors confirm that the board densities were found to have major influence on the machinability characteristics of the panel. Recently Davim et al [9] presents the study of surface roughness aspect in milling MDF. In his study, the surface roughness in milling decreases with an increase of spindle speed and with an increase with feed rate.

Yang et al [10] have used Taguchi method to find the optimal cutting parameters for turning opertions.Choudhury and El-Baradie [11] have used response surface methodology for predicting surface roughness of high strength steel. Thomas et al [12] used a full factorial design to investigate the effect of cutting tool parameter on the surface toughness of carbon steel. Nemli et al [13] have investigated the effects of various parameters on the surface roughness of the particleboard. According to his study, density is one of the major factors influencing the surface roughness of particleboard. Tabarsa et al [14] have concluded that increasing press time and moisture content of the particleboard decreases the surface roughness. Prakash et al [15] have formulated a mathematical model for the prediction of surface roughness in drilling Medium Density Fiber board (MDF) panel. Their results show that the surface roughness increases with decrease of spindle speed and increase of feedrate.

In conventional multifactor experiments, the optimization is usually carried out by varying a single factor while keeping all other factors at a specific set of conditions. Moreover, this approach is time consuming and ignores the combined interactions between the parameters. To solve this problem, response surface methodology can be employed as an interesting strategy to implement process conditions, which drive to optimal response by performing a minimum number of experiments. RSM is combination of mathematical and stastical techniques used for, developing, improving and optimizing the parameters for the required output response.

In the present work, an attempt has been made to employ Box-Behnken design using response surface methodology for optimizing the key influencing parameters on the surface roughness $R a, R z$ in MDF composites.

\section{EXPERIMENTAL WORK}

\section{A. Material}

The material used for the present investigation is MDF board. The boards are supplied by ASIS, India, which are manufactured by them. These boards are commercially available and used for furniture industry. The important properties of the board as per ISO 12406 are given in Table 1.

Table 1. Mechanical and Physical Properties of MDF board tested

\begin{tabular}{|c|c|c|c|c|}
\hline $\begin{array}{r}\text { Tensile } \\
\text { strength } \\
\mathrm{N} / \mathrm{mm}^{2}\end{array}$ & $\begin{array}{c}\text { Modulus } \\
\text { Of } \\
\text { rupture } \\
\mathrm{N} / \mathrm{mm}^{2}\end{array}$ & $\begin{array}{c}\text { Elasticity } \\
\text { modulus } \\
\mathrm{N} / \mathrm{mm}^{2}\end{array}$ & Humidity\%$\%$ & $\begin{array}{l}\text { Density } \\
\mathrm{Kg} / \mathrm{mm}^{3}\end{array}$ \\
\hline 0.8 & 28 & 2800 & $5-8$ & $600-900$ \\
\hline
\end{tabular}

\section{B. Equipment}

The drilling tests were performed on ARIX VMC 100 machining centre with the following specifications presented in Table 2

Table 2. Machine Specification

\begin{tabular}{|l|l|l|}
\hline \multirow{4}{*}{ Capacity } & X axis & $100 \mathrm{~mm}$ \\
\cline { 2 - 3 } & Y axis & $500 \mathrm{~mm}$ \\
\cline { 2 - 3 } & Z axis & $500 \mathrm{~mm}$ \\
\hline \multirow{3}{*}{ Table } & Size & $1270 \times 330 \mathrm{~mm}$ \\
\cline { 2 - 3 } & T-Slots & $16 \times 3 \mathrm{~mm}$ \\
\hline \multirow{3}{*}{ Spindle } & Tool mounting & $1 S 040$ \\
\cline { 2 - 3 } & Speed & $60 / 5000 \mathrm{rpm}$ \\
\hline \multirow{2}{*}{ Feed rates } & Feed rate & $4000 \mathrm{~mm} / \mathrm{min}$ \\
\cline { 2 - 3 } & Rapid traverse & $4000 \mathrm{~mm} / \mathrm{min}$ \\
\hline
\end{tabular}

The drill bit used in the investigation is 'Brad and spur' carbide type, having diameters of 4,8 and 12 $\mathrm{mm}$. The experimental setup is presented in Fig, 1 .

The experimental design is used to conduct experiments with less number of observations. They constitute a systematic method concerning the planning 
of experiments, collection and analysis of data with near optimum use of available sources.

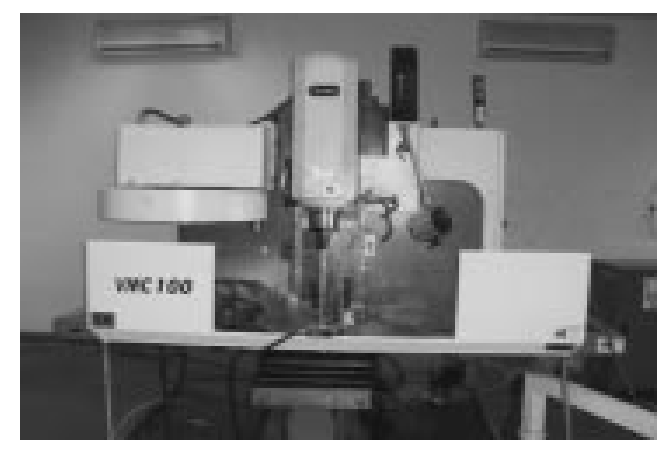

Fig 1 Experimental setup

The factors, considered for experimentation and analysis are cutting speed, feed rate and drill diameter.

\section{RESPONSE SURFACE METHODOLOGY}

RSM is the collection of mathematical and statistical techniques that are useful for the modeling and analysis of problems in which a response of interest is influenced by several variables and the objective is to optimize this response[13].RSM also quantifies the relationship between the controllable input parameters and the obtained response surfaces[14-15].The design procedure of RSM is as follows [16-17]

1. Designing a series of experiments for adequate and reliable measurements of the response of interest.

2. Developing a mathematical model of the response surface with the best fitting.

3. Finding the optimal set of experimental parameters that produce a maximum or minimum value of the response.

4. Representing the interaction effects of the parameters through contour plots and three dimensional plots.

\section{THE BOX-BEHNKEN DESIGN AND THE RESPONSE EQUATION}

The Box-Behnken design is an independent quadratic design, that does not contain an embedded factorial or fractional factorial design [18]. In this design the treatment combinations are at the midpoints of edges of the process space and at the center. These designs are rotatable (or near rotatable) and require 3 levels of each factor. Fig.2 illustrates a Box-Behnken design for three factors[22]. For three factors, the Box-Behnken design offers some advantages in requiring a fewer number of runs.

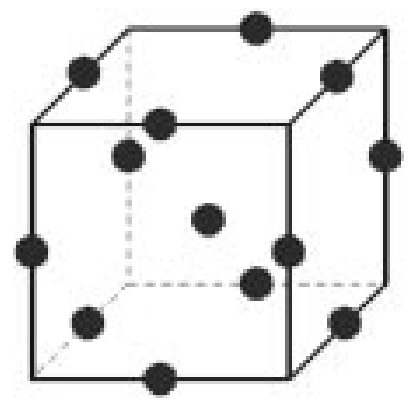

Fig.2 Box-Behnken Design for Three Factors

The detailed design is given in Table 3 .

Table.3 Behnken Design

\begin{tabular}{|l|l|l|l|}
\hline Factors & 3 & Replicates & 1 \\
\hline Base run & 17 & Total runs & 17 \\
\hline Base blocks & 1 & Total blocks & 1 \\
\hline Centre points & & 3 & \\
\hline
\end{tabular}

The experiments were carried out as per RSM Box-Behnken experimental design [19]. A set consisting of 17 experiments were conducted for developing the mathematical model for surface roughness in drilling MDF composite by carbide drills. The input parameters and their levels chosen for this work are given in Table 4.

Table.4 Experimental design level of chosen parameters

\begin{tabular}{|l|c|c|c|}
\hline Parameters & \multicolumn{3}{|c|}{ Levels in Box Behnken Design } \\
\hline Coded level & Low $(-1)$ & Middle (0) & High $(+1)$ \\
\hline Feed(mm/min) & 100 & 300 & 500 \\
\hline Speed $(\mathrm{rpm})$ & 1000 & 3000 & 5000 \\
\hline Drill dia(mm) & 4 & 8 & 12 \\
\hline
\end{tabular}

There are various methods to evaluate surface roughness of composite panels, which include acoustic emission, pneumatic, laser and stylus [18, 23]. The stylus techniques is extensively used and well established to quantify surface roughness of industrial metal and plastic parts. The main advantage of stylus 
method is that it uses standard numerical parameters and profile of surface. The surface roughness of MDF has been measured by using Taylor Hobson surface roughness measuring instrument, shown in Fig 5 . The surface roughness used in this study is the arithmetic mean average surface roughness $\left(R_{a}\right)$, and mean peak to valley height $(\mathrm{Rz})$ which is widely used in the industry.

The condition with real values, coded values of parameters and experimental results are presented in Table 5.The corresponding surface roughness value plots are shown in Fig 6 and 7 which is measuring using Taylor Hobson surface roughness measuring instrument.

The mathematical expression of relationship of the surface roughness parameter with the three variables (feedrate, speed, drill diameter) is shown below as in terms of actual factors. These equations make it possible to predict the surface roughness.[19-21]

$\mathrm{Ra}$, as shown in Fig 3, can be expressed by the following mathematical relationship and presented as

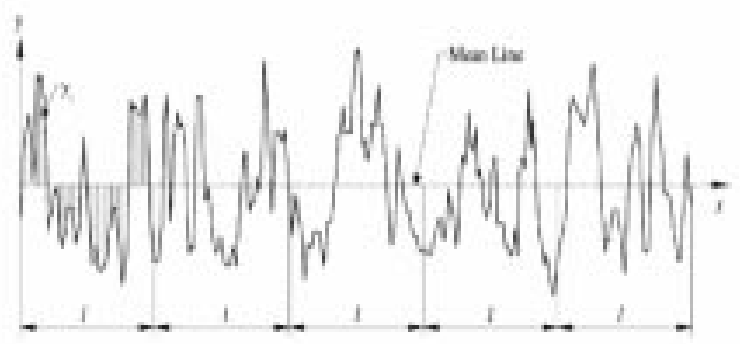

Fig 3. Definition of the arithmetic average height parameter $\left(R_{a}\right)$

$$
\begin{array}{r}
R_{a}=7.47+1.92 E-3 * f-1.51 E-4 * N+0.33 \\
* d+2.13 E \\
-5 * f F^{2}+6.15 E-8 * N^{2}-0.012 * d^{2} \\
-1.63 E-6 f * N-1.56 E-5 * f * d-5 E-6 \\
* N * d
\end{array}
$$

Where $\mathrm{N}$ - speed in rpm, F-Feed in $\mathrm{mm} / \mathrm{min}$, D-drill diameter in $\mathrm{mm}$

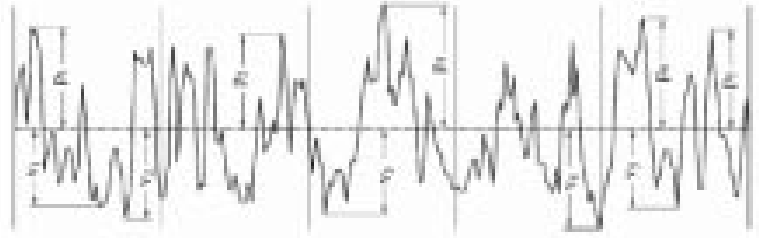

Fig 4. Definition of the ten-point height average parameter $\left(R_{\mathbf{z}}\right)$

$\mathrm{Rz}$, as shown in Fig 4 can be expressed by the following mathematical relationship and presented as

$$
\begin{array}{r}
R z=32.49+0.10 * f+4.12 E-05 * N+2.04 \\
* d-4.2 * f^{2}-4.9 E-0.7 * N^{2} \\
-0.088 * d^{2}-6.61 * f * N-0.00229 * f * \\
d+0.003 * N * f
\end{array}
$$



Fig 5-Equipment used for measuring $\mathrm{Ra}$ and $\mathrm{Rz}$

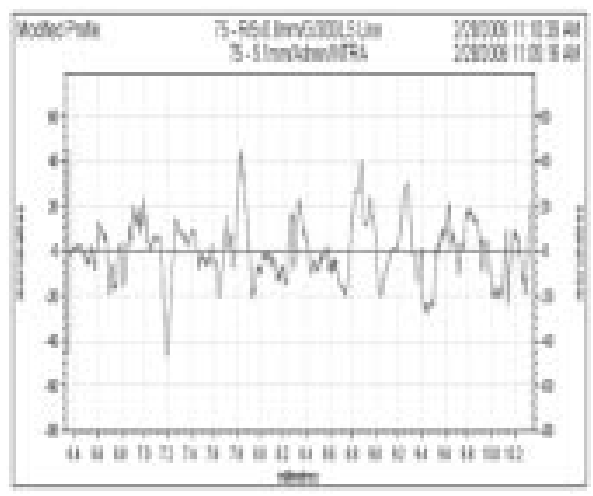

Fig 6. Surface Roughness plot at $N=3000 \mathrm{rpm}$ $f=300 \mathrm{~mm} / \mathrm{min}$ diameter $=8 \mathrm{~mm}$ 


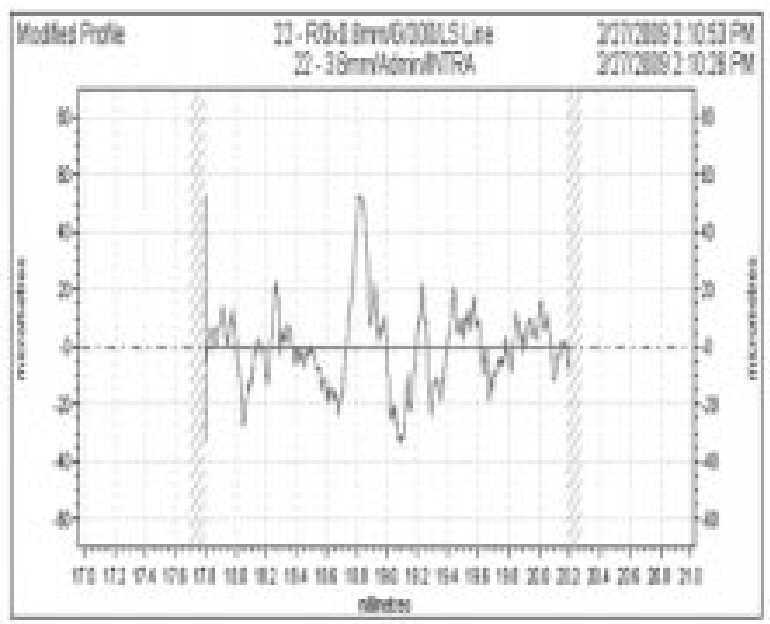

Fig 7-Surface Roughness plot at $n=1000 \mathrm{rpm}$, $f=300 / \mathrm{mm} / \mathrm{min}$, diameter $=4 \mathrm{~mm}$

The PRESS values for Average surface roughness $(\mathrm{Ra})$ and Mean peak to valley height $(\mathrm{Rz})$ are 2.70 (low value) and 166.58 (low value) shows that the quadratic model is the best fitted model for the prediction of surface roughness parameters in drilling MDF panels. Table 7 and Table 8 shows the ANOVA results for the quadratic equation of surface roughness parameters ( $\mathrm{Ra}$ and $\mathrm{Rz}$ ).

Table 5. Box-Behnken design consisting of experiments for the study of three experimental factors in coded and actual levels with experimental and predicted values for surface roughness (Ra and $\mathbf{R z}$ )

\begin{tabular}{|c|c|c|c|c|c|c|c|c|c|}
\hline \multirow{3}{*}{ Run } & \multirow{2}{*}{ Drill type } & \multirow{2}{*}{\multicolumn{3}{|c|}{ Coded values }} & \multirow{2}{*}{\multicolumn{3}{|c|}{ Original values }} & \multicolumn{2}{|c|}{ Output Response } \\
\hline & & & & & & & & \multirow{2}{*}{$\frac{\mathbf{R a}(\mu \mathrm{m})^{*}}{\text { Experiment }}$} & \multirow{2}{*}{$\frac{\mathbf{R z}(\mu \mathrm{m})^{*}}{\text { Experiment }}$} \\
\hline & & $A$ & B & $\mathrm{C}$ & Feed & Speed & Dia & & \\
\hline 1 & & -1 & -1 & 0 & 100 & 1000 & 8 & 9.25 & 52.76 \\
\hline 2 & & 1 & -1 & 0 & 500 & 1000 & 8 & 14.57 & 77.35 \\
\hline 3 & & -1 & 1 & 0 & 100 & 5000 & 8 & 9.54 & 52.05 \\
\hline 4 & & 1 & 1 & 0 & 500 & 5000 & 8 & 12.24 & 66.06 \\
\hline 5 & & -1 & 0 & -1 & 100 & 3000 & 4 & 8.56 & 47.13 \\
\hline 6 & & 1 & 0 & -1 & 500 & 3000 & 4 & 12.33 & 68.06 \\
\hline 7 & & -1 & 0 & 1 & 100 & 3000 & 12 & 9.61 & 60.83 \\
\hline 8 & & 1 & 0 & 1 & 500 & 3000 & 12 & 13.33 & 74.42 \\
\hline 9 & & 0 & -1 & -1 & 300 & 1000 & 4 & 10.65 & 65.72 \\
\hline 10 & & 0 & 1 & -1 & 300 & 5000 & 4 & 9.24 & 51.26 \\
\hline 11 & & 0 & -1 & 1 & 300 & 1000 & 12 & 11.54 & 67.12 \\
\hline 12 & & 0 & 1 & 1 & 300 & 5000 & 12 & 9.97 & 65.19 \\
\hline 13 & & 0 & 0 & 0 & 300 & 3000 & 8 & 10.3 & 65.72 \\
\hline 14 & & 0 & 0 & 0 & 300 & 3000 & 8 & 10.3 & 65.72 \\
\hline 15 & & 0 & 0 & 0 & 300 & 3000 & 8 & 10.3 & 65.72 \\
\hline 16 & & 0 & 0 & 0 & 300 & 3000 & 8 & 10.3 & 65.72 \\
\hline 17 & & 0 & 0 & 0 & 300 & 3000 & 8 & 10.3 & 65.72 \\
\hline
\end{tabular}


Prakash et al : Evaluation of Surface Roughness Parameters...

* Average of three results

The model summary statistics of Average surface roughness $(\mathrm{Ra})$ and Mean peak to valley height $(\mathrm{Rz})$ of drilling MDF panels are illustrated in Table 6

\section{RESULTS AND DISCUSSION}

A. ANOVA and response plots

The analysis of variance (ANOVA) was applied to study the effect of the input parameters on the surface roughness. Table 6

Table 6 Model summary statistics of Average surface roughness (Ra) and Mean peak to valley height (Rz) of drilling MDF panel

\begin{tabular}{|c|c|c|c|c|c|c|}
\hline \multirow{2}{*}{ Source } & \multicolumn{3}{|c|}{ Average surface roughness(Ra) } & \multicolumn{3}{c|}{ Mean peak to valley height (Rz) } \\
\cline { 2 - 7 } & Linear & 2FI & Quadratic & Linear & 2FI & Quadratic \\
\hline SD & 0.64 & 0.61 & 0.16 & 3.15 & 2.20 & 1.02 \\
\hline$R^{2}$ & 0.8661 & 0.9089 & 0.9958 & 0.8775 & 0.9540 & 0.9931 \\
\hline Adj R & 0.8352 & 0.8542 & 0.9904 & 0.8493 & 0.9265 & 0.9842 \\
\hline Pred R & 0.7414 & 0.6217 & 0.9330 & 0.7698 & 0.8727 & 0.8895 \\
\hline PRESS & 10.42 & 15.25 & $\mathbf{2 . 7 0}$ & 242.80 & 134.26 & 116.58 \\
\hline
\end{tabular}

Table.7.Results of ANOVA for the quadratic equation of surface roughness (Ra) in drilling MDF

\begin{tabular}{|l|c|c|c|c|}
\hline \multicolumn{1}{|c|}{ Source } & Degree of Freedom & Sum of squares & Mean square & F value \\
\hline Model & 9 & 40.13 & 4.43 & 185.05 \\
\hline $\mathrm{A}($ Feed $)$ & 1 & 30.07 & 30.07 & 1247.90 \\
\hline B(Speed) & 1 & 3.15 & 3.15 & 130.73 \\
\hline C (Dia) & 1 & 1.68 & 1.68 & 69.87 \\
\hline $\mathrm{A}^{2}$ & 1 & 3.07 & 3.07 & 127.36 \\
\hline $\mathrm{B}^{2}$ & 1 & 0.26 & 0.26 & 10.60 \\
\hline $\mathrm{C}^{2}$ & 1 & 0.16 & 0.16 & 6.73 \\
\hline $\mathrm{AB}$ & 1 & 1.72 & 1.72 & 71.22 \\
\hline AC & 1 & $6.250 \mathrm{E}-004$ & $6.250 \mathrm{E}-004$ & 0.026 \\
\hline BC & 1 & $6.400 \mathrm{E}-003$ & $6.400 \mathrm{E}-003$ & 0.27 \\
\hline Residual & 7 & 0.17 & 0.17 & \\
\hline Lack of fit & 3 & 0.17 & 0.17 & \\
\hline Pure Error & 4 & 0.000 & 0.000 & \\
\hline Car Total & 16 & 40.30 & 40.30 & \\
\hline
\end{tabular}


Table.8. Results of ANOVA for the quadratic equation of surface roughness (Rz) in drilling MDF

\begin{tabular}{|l|c|c|c|c|}
\hline \multicolumn{1}{|c|}{ Source } & Degree of Freedom & Sum of squares & Mean square & F value \\
\hline Model & 9 & 1047.52 & 116.39 & 111.82 \\
\hline $\mathrm{A}($ Feed $)$ & 1 & 668.32 & 668.32 & 642.05 \\
\hline $\mathrm{B}($ Speed) & 1 & 100.75 & 100.75 & 96.79 \\
\hline $\mathrm{C}$ (Dia) & 1 & 156.56 & 156.56 & 150.40 \\
\hline $\mathrm{A}^{2}$ & 1 & 12.01 & 12.01 & 11.54 \\
\hline $\mathrm{B}^{2}$ & 1 & 16.44 & 16.44 & 15.80 \\
\hline $\mathrm{C}^{2}$ & 1 & 8.51 & 8.51 & 8.17 \\
\hline $\mathrm{AB}$ & 1 & 27.98 & 27.98 & 26.88 \\
\hline $\mathrm{AC}$ & 1 & 13.47 & 13.47 & 12.94 \\
\hline $\mathrm{BC}$ & 1 & 39.25 & 39.25 & 37.71 \\
\hline Residual & 7 & 7.29 & 1.04 & \\
\hline Lack of fit & 3 & 7.29 & 2.43 & \\
\hline Pure Error & 4 & 0.000 & 0.000 & \\
\hline Car Total & 16 & 1054.81 & & \\
\hline
\end{tabular}

gives the model statistics. It reveals that the quadratic model is the best-suggested model. So, for further analysis this model was used. Table $7 \& 8$ gives the ANOVA results for the quadratic equation for the surface roughness $R a$ and $R z$. ANOVA is commonly used to summarize the test for significance on individual model coefficients. The value Of "Prob>F" for model indicates that the model terms are significant, which is desirable as indicated that the terms in the model have significant effect on the response. The model $F$ value of $185.05(\mathrm{Ra})$ and $111.82(\mathrm{Rz})$ implies that the model is siginificant.There is only a $0.01 \%$ chance that the "Model F value" this large could occur due to noise.

Table 9 gives the regression statistics. The coefficient of determination $R^{2}$ is used to decide whether a regression model is appropriate. The coefficient of determination $R^{2}$ provides an exact match if it is 1 and if the residual increases $R^{2}$ decreases in the range from 1 to 0 .

As the number of variables increase, the residuals decrease, so that the co efficient of determination $\mathrm{R}^{2}$, increases its value. So, to obtain a more precise regression model judgment, the co efficient of determination $R^{2}$ is adjusted for the degrees of freedom.

Table 9 Regression statistics

\begin{tabular}{|l|c|c|}
\hline \multicolumn{1}{|c|}{ Statistical values } & $\mathbf{R a}$ & $\mathbf{R z}$ \\
\hline Standard Deviation & 0.16 & 1.02 \\
\hline Mean & 10.73 & 63.33 \\
\hline CV(Coefficient of variation) & 1.45 & 1.61 \\
\hline PRESS & 2.7 & 116.58 \\
\hline $\mathrm{R}^{2}$ & 0.9958 & 0.9931 \\
\hline Adjusted $\mathrm{R}^{2}$ & 0.9904 & 0.9842 \\
\hline Predicted $\mathrm{R}^{2}$ & 0.9330 & 0.8895 \\
\hline Adequate precision & 51.016 & 38.566 \\
\hline
\end{tabular}

Adj $R^{2}$ is used for comparing the residuals per unit degree of freedom. Adequate precision compares the range of the predicted values at the design points to the average prediction error. It is a measure of the 
signal to noise ratio. Ratio greater than 4 indicates adequate model discrimination. In this particular case, it is 51.016 for $\mathrm{Ra}$ and 38.566 for $\mathrm{Rz}$ which is well above 4 . So the model can be used to navigate the response space.

The adequacy of the model has also been investigated by the examination of residuals [15].The residuals, which are the difference between the respective observed responses and the predicted responses, are examined using the normal probability plots of the residuals and the plot of residuals versus predicted response. The normal probality plot is represented in Fig.8.

The normal probability plot is a graphical technique for assessing whether or not a data set is approximately normally distributed. The data are plotted against a theoretical normal distribution in such a way that the points should form an approximate straight line.

In these designs, the probability plots show a strongly linear pattern. This is verified by the correlation coefficient of 0.98 of the line fit to the probability plot. The fact that the points in the lower and upper extremes of the plot do not deviate significantly from the straight-line pattern indicates that there are not any significant outliers (relative to a normal distribution).

In this case, it can be concluded that the normal distribution provides an excellent model for the data.

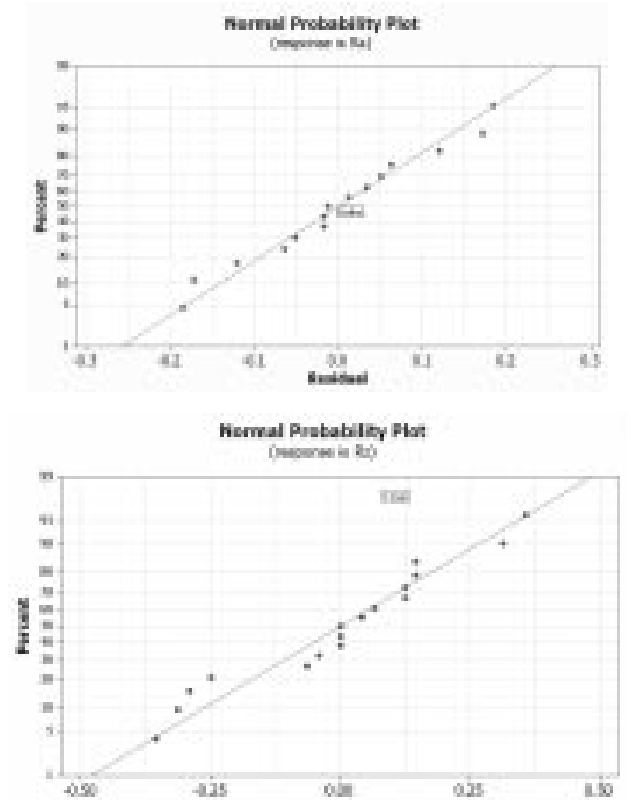

Fig.8 Normal probability plot of residuals for surface roughness $(\mathrm{Ra})$
It revealed that the residuals generally fall on a straight line implying that the errors are distributed normally

In addition, the model gives $\mathrm{R}^{2}$ values of 0.995 and 0.993 and the adjusted $R^{2}$ values of 0.99 and 0.984 for $\mathrm{Ra}$ and $\mathrm{Rz}$ respectively. These values confirm that the equation model is highly reliable. This indicates also that the model terms are significant at $95 \%$ of the probability level.

Fig 9 shows the standardised residuals vs predicted surface roughness plot. The general impression is that the plot should be a random scatter, suggesting that the variance of original observations is constant for the all values of the response.
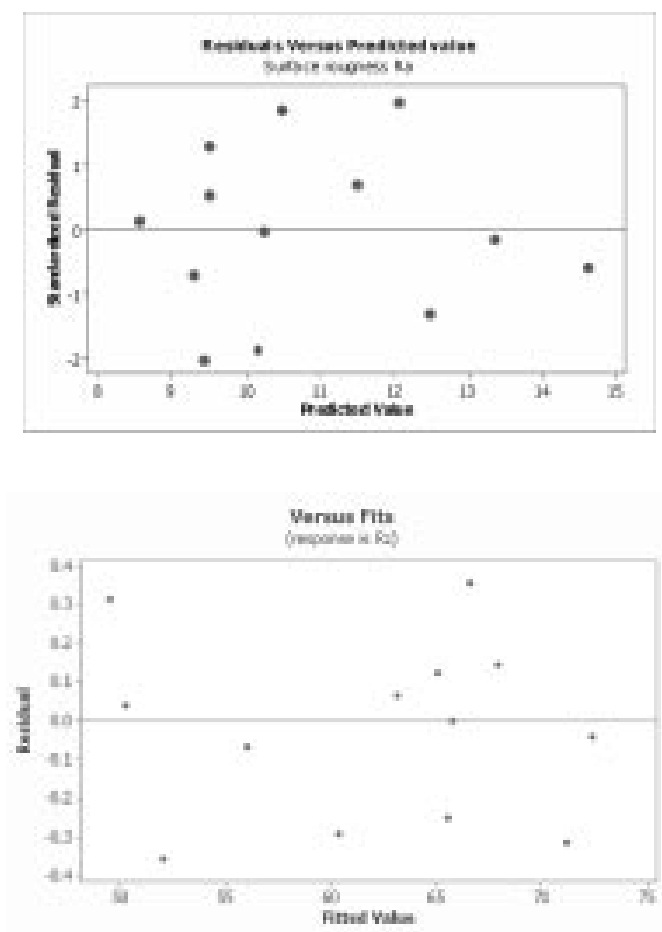

Fig.9 Predicted surface roughness and standardized esidual plots

Generally, it is important to confirm the fitted model to make sure that it gives sufficient approximation to the actual test.

\subsection{Effect of parameters on surface roughness}

Using experimental design, the combined effects of three variables can be predicted which is difficult to observe in conventional methods. The effect of variables on surface roughness are shown in Figs 10-11.These figures show the $3 \mathrm{D}$ response plots of 
interaction between varying feedrate and speed on surface roughness where the diameter is kept at a constant value $(4 \mathrm{~mm})$.

As the models are adequate these 3D surface plots can be used for estimating the surface roughness values for any suitable combination of the input parameters namely feedrate, speed and drill diameter [16]

Equations discussed above gives the prediction model for surface roughness in terms of actual factors. These response contours can help in the prediction of surface roughness at any zone of the experimental domain [12].

It is clear from these figures that the surface roughness reduces with increase of speed. It also indicates that the surface roughness increases with increase of feed. Fig.11 shows the 3D response surface plot which reveals the effect of drill diameter with respect to feed rate. As Fig 10 shows, contrary to the feed, the surface roughness increases with decrease with speed.
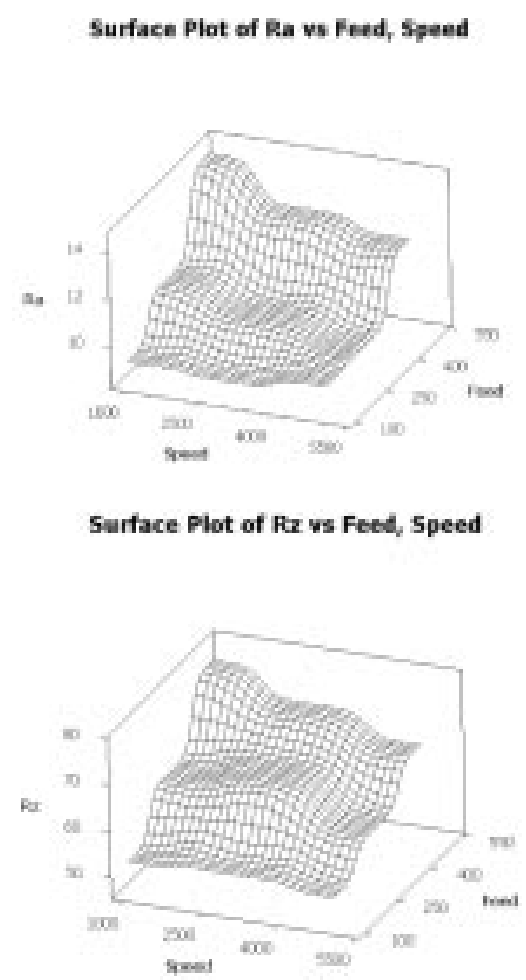

Fig.10 3D response surface plots for speed vs feed at diameter $4 \mathrm{~mm}$

\section{CONFIRMATION TEST}

The effectiveness of the model has been checked by the validation with experimental values. In order to verify the adequacy of the model developed, the confirmation test was so chosen that they be with in the range of the levels defined previously The predicted value and the associated experimental values are compared and the percentage error was calculated. (Table 10)

The error percentage is with in permissible limits. So the response equations for the surface roughness evolved through RSM-Box-Behnken can be successfully predict the surface roughness values for any combination of the feed rate, speed and drill diameter with in the range of experimentation conducted. The experimental results have been validated by asserting that the predicted values are very close to each other and hence the developed models are suitable.

Surface Plot of Ra vs Feed, Diameter

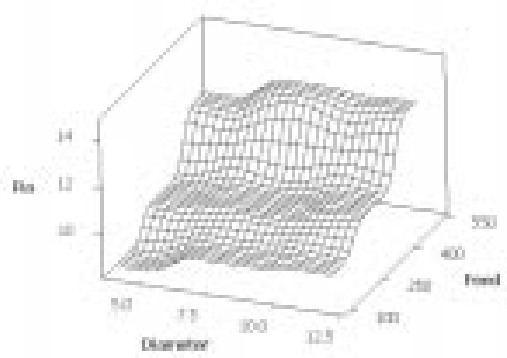

Surface Plot of Rz vs Feed, Diameter

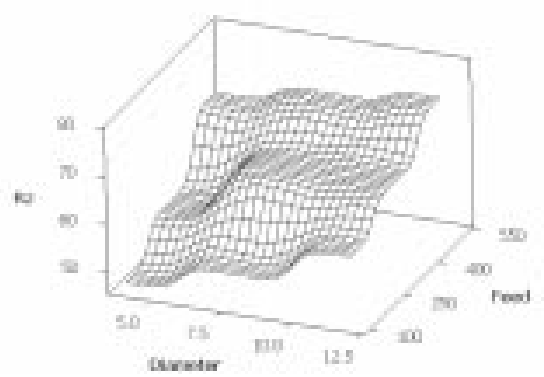

Fig.11 3D response surface plots for feed vs diameter at speed $1000 \mathrm{rpm}$ 
Table.10.Confirmation test data (Experimental vs RSM model)

\begin{tabular}{|l|c|}
\hline \multicolumn{2}{|l|}{ Test parameter (input parameters) } \\
\hline Speed(rpm) & 1000 \\
\hline Feedrate(mm/min) & 300 \\
\hline Drill diameter(mm) & 10 \\
\hline Surface roughness Ra $(\mu \mathrm{m})$ (outputresponse) \\
\hline Experimental $(\mu \mathrm{m})$ & 11.12 \\
\hline RSM predicted $(\mu \mathrm{m})$ & 11.35 \\
\hline Error $(\%)$ & $2.06 \%$ \\
\hline Surface roughness Rz ( $\mu$ m) (output response) \\
\hline Experimental $(\% \mathrm{~m})$ & 66.88 \\
\hline RSM predicted $(\% \mathrm{~m})$ & 65.12 \\
\hline Error(\%) & $2.631 \%$ \\
\hline
\end{tabular}

\section{CONCLUSIONS}

In this paper, RSM (Box-Behnken design) has been used to determine the surface roughness attained by the drilling of MDF panels for various input parameters namely feed rate, speed and drill diameter. An RSM model can successfully relate the above process parameters with the response, surface roughness.

The following conclusions are drawn from experimental results during drilling of MDF board using 4, 8 and $12 \mathrm{~mm}$ Brad and spur carbide drill bits under different cutting conditions:

1. The quadratic factor interaction model for surface roughness has been developed using Box-Behnken RSM experimental design method.

2. The surface roughness, both $R a$ and $R z$ increases with increasing feed but decreases with increasing spindle speed

3. The established equations clearly show that the feed is the factor which influences surface roughness more followed by spindle speed.

4. The predicted and the measured values are close to each other which indicate that the developed surface roughness prediction model can be effectively used for predicting the surface roughness during drilling of MDF panel with more than $95 \%$ confidence level.

\section{REFERENCES}

[1] Aguilera, A., Meausoone, P.J., Martin, P., 2000. Wood material in?uence in routing operations: the MDF case. Holz als RohWerkstoff 58, 278-283.

[2] Irle, M., Loxton, C., 1996. Manufacture and use of panel products in the UK. J. Inst. Wood Science. 14, 21-26.

[3] Neese JL, Reeb JE, Funck JW. Relating traditional surface roughness measures to glue-bond quality in plywood. Forest Products Journal 2004;54(1):67-73.

[4] H.A.Stewart, "High temperature halogenations of tungsten carbide-cobalt tool material when machining MDF", Forest Products Journal, 42 (10): 27-31, 1992.

[5] Engin S, Altintas Y, Amara FB (2000) Mechanics of routing medium density fiberboard. Forest Prod $\mathrm{J}$ 50(9):65-69

[6] Dippon, J, Ren, H., Amara, F.B., Altintas,Y Orthogonal-cutting mechanics of medium density fiberboards, For. Prod. J. 50 (2000) 25-30.

[7] Philbin P, Gordon S (2006) Recent research on the machining of wood-based composite materials. International Journal of Machining and Machinability of Materials 1(2):186-201

[8] Lin R. J, VanHouts. J, Bhattacharyya. D, Machinability investigations of medium-density fiberboard, Holzforschung, 60 (2006) 71-77.

[9] J. Paulo Davim \& V. C. Clemente \& Sérgio Silva Surface roughness aspects in milling MDF (medium density fiberboard) Int J Adv Manuf Technol DOI 10.1007/s00170-007-1318-z

[10] Yang.W.H.,Tang,Y.S.,(1998),Design optimization of cutting parameters for turning operations based on Taguchi method.J.Mater.Process.Technolo,84,112-119

[11] Choudhury,I.A., El.Baradie,M.A.,(1997), Surface roughness predication in the turning of high strength steel by factorial design of experiments, J.Mater.Process.Technol,67,55-61

[12] Thomas,M., Bcauchamp, $\quad$ Y.,Youssef,Y.A., Masounave,J.,(1997).An experimental design for surface roughness and built up edge formation in lathe dry turning, Int.J.Qual.Sci.2(3),167-180

[13] Gokay Nemli, Ismail Aydin, Emir Zekovic, "Evaluation of some of the properties of particle board as function of manufacturing parameters, Journal of Materials and Design vol 28(2007) pp 1169-1176.

[14] Taghi Tabarsa,Alireza Ashori, Maria Gholamzadeh, "Evaluation of surface roughness and mechanical 
properties of particle board panels made from bagesse", Journal of composites, Part B (2011) doi 10.1016/j.compositesb.2010.12.018.

[15] S.Prakash,K.PalaniKumar, "Modeling for prediction of surface roughness in drilling MDF panels using response surface methodology",Journal of Composite materials, 2010, doi:10.1177/0021998310385026

[16] Cochran WG,Cox GM(1992)Experimental designs,Wiley,New York.

[17] Jae-seob,Kwak,2005,Application of Taguchi and response surface methodologies for geometric error in surface grinding process.Int.J.Mach.Tools Manuf.45,327-334

[18] Montogomery,C.,1997,Design and Analysis of experiments, $4^{\text {th }}$ ed.Wiley.New york

[19] Noordin,M.Y.,Venkatesh,V.C.,Sharif., Elting,S.,Abdullah,A.,2004,Application of response surface methodology in describing the performance of coated tools when turning AISI 1045 steel ,J.Mater .Process Technolo, 145,46-58

[20] Gunaraj,V.,Murgan,N.,1999.Application of response surface methodology for predicting weld bead quality in submerged arc welding of pipes.,J.Mater Process.Tehnolo.88(1-3), 15,pp,266-275.

[21] G. E. P. Box and N. R. Draper in "Empirical Model Building and Response Surfaces," John Wiley and Sons, New York, 1987

[22] George Box, Donald Behnken, "Some new three level designs for the study of quantitative variables", Technometrics, Volume 2, pages 455-475, 1960.

[23] Murat Kilic.,Salim Hiziroglu.,Erol Burdurlu,2006 Effect of machining on surface roughness of wood. International Journal of Building and Environment, 41 pp 1074-1078 